\title{
3D atom probe analysis of quantum well and quantum dot materials
}

\author{
A. Cerezo*, L. Chang****, P.H. Clifton***, M.J. Galtrey**, S.S.A. Gerstl***, C.J. Humphreys**, \\ M. Müller*, R.A. Oliver**, G.D.W. Smith*, Y.H. Wu****. \\ * Department of Materials, University of Oxford, Parks Road, Oxford, OX1 3PH, UK \\ ** Department of Materials Science and Metallurgy, University of Cambridge, Pembroke Street, \\ Cambridge, CB2 3QZ, UK \\ *** Imago Scientific Instruments, 5500 Nobel Drive, Madison, WI 53711, USA \\ **** Department of Materials Science and Engineering, National Chiao Tung University, Hsinchu, \\ Taiwan 300
}

The use of the 3D atom probe (3DAP) for atomic-scale analysis of metallic alloys is well known [1], but specimen shape and conductivity requirements have typically limited the application of the technique for the analysis of semiconductor materials. Kellogg and Tsong developed the use of laser pulsing to allow analysis of less conductive materials [2], and this permitted the first 3DAP analysis of multiple quantum well (MQW) materials [3]. With the advent of focussed-ion beam (FIB) lift-out techniques for specimen preparation, $[4,5]$ and modern high-speed lasers, the 3DAP has developed into a powerful technique for analysis of semiconductor nanostructures [6].

Optoelectronic devices based on III-nitride quantum well (QW) structures exhibit bright photoluminescence despite very high dislocation densities. High resolution electron microscopy (HREM) has often shown significant inhomogeneous strain contrast in the InGaN quantum wells, suggesting the presence of In-rich clusters which could act as exciton localisation centres, reducing the deleterious effects of dislocations in the material [7]. However, if images are taken using low electron beam doses no such contrast is observed, implying that the "clusters" are an artefact caused by electron beam exposure [8]. 3DAP analysis provides an alternative method for identifying whether or not In clusters exist in the QWs. Figure 1 shows 3DAP data from a $\operatorname{In}_{x} \mathrm{Ga}_{1-\mathrm{x}} \mathrm{N} / \mathrm{GaN} \mathrm{MQW}$ sample, similar to those studied with HREM by Smeeton et al. [8], grown by metallo-organic vapour-phase epitaxy. The isosurfaces show no sign of In levels reaching $\mathrm{x}=0.7$ as has been suggested in the literature, and frequency distribution analysis of the wells shows no evidence of non-random composition fluctuations [9].

Nanometre-size self-assembled quantum dots (QDs) are important for long wavelength lasers in optical communications. QDs are formed by Stranski-Krastanov growth, after deposition of a very thin wetting layer, and can form complex structures as shown in Figure 2(a). Optimisation of these materials depends on knowledge of the chemical variations within the QDs, but the complex 3-D morphology makes it hard to obtain this information by conventional means. For example, HREM images suggested non-uniform composition profiles in QDs [10], but accurate characterisation is confused by the 2D projection and uncertainty of where the QD lies within the foil. 3DAP analysis is able to provide crucial composition and morphological information to feed into models of the QD growth and properties. Figure 2 shows the 3DAP analysis of a MBE grown structure with InAs QDs embedded in GaAs, where the individual dots and wetting layers are clearly seen. We believe this is the first 3DAP analysis of a quantum dot material. Initial results from this work show significant variations in the In concentration within the QDs. 
(a)

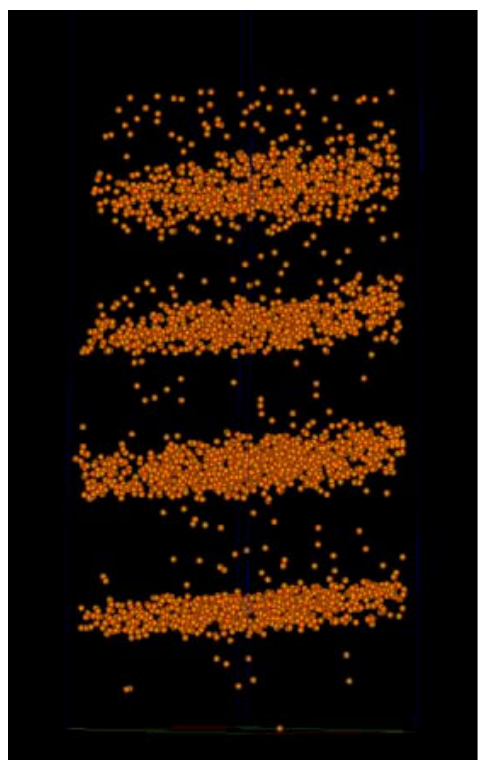

(b)

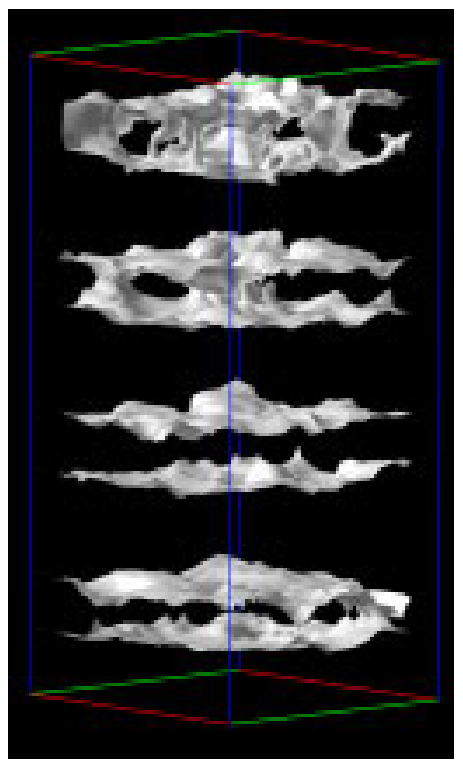

(c)

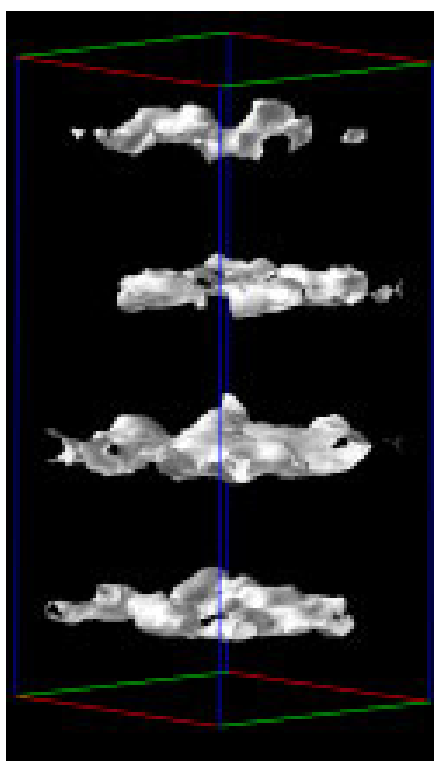

FIG. 1. 3DAP analysis of $\operatorname{In}_{x} \mathrm{Ga}_{1-\mathrm{x}} \mathrm{N} / \mathrm{GaN}$ MQW structure: (a) In atom map, showing $2.4 \mathrm{~nm}$ thick InGaN QWs; (b) and (c) iso-concentration surfaces drawn at In levels of (b) $\mathrm{x}=0.06$ and (c) $\mathrm{x}=0.16$.

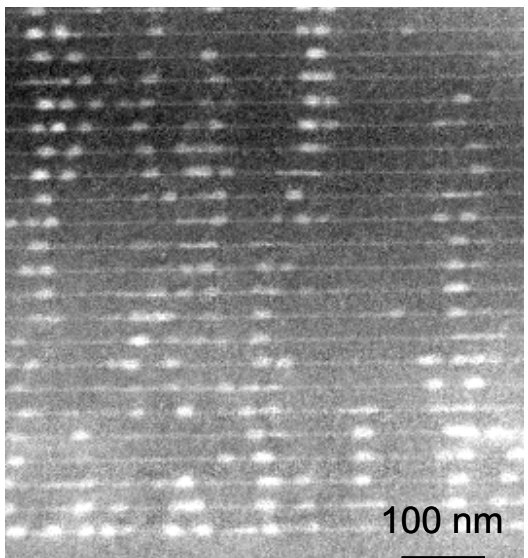

(a)

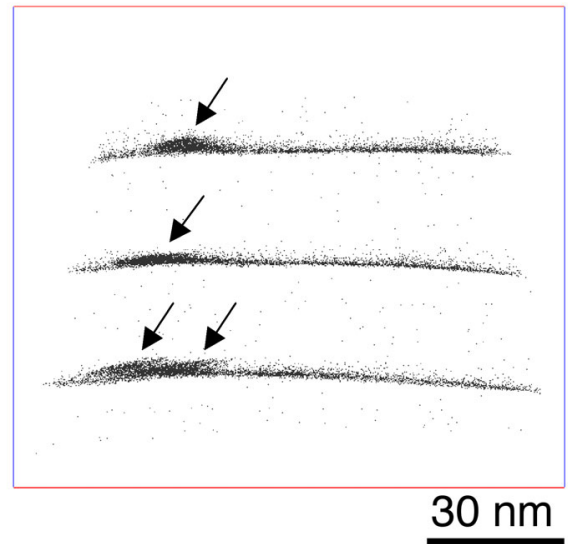

(b)

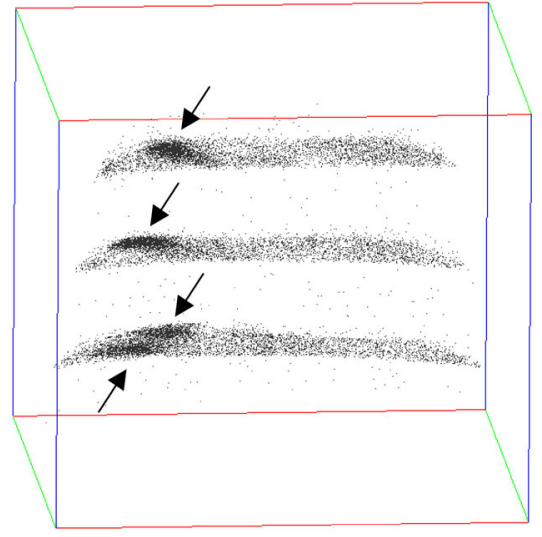

(c)

FIG. 2. (a) Dark field STEM image of InAs QDs embedded in GaAs, where InAs dots and layers appear in light contrast. (b) and (c) In atom maps from a 30nm slice through the 3DAP analysis of the same material viewed from the side (b) and tilted (c) showing the InAs QDs (arrowed).

\section{References}

[1] M.K. Miller, Atom probe tomography, Plenum, New York, 2000.

[2] Kellogg, G.L. and Tsong, T.T. (1980). J Appl Phys 511184.

[3] J.A. Liddle et al., Appl. Phys. Lett. 54 (1989) 1555.

[4] M.K. Miller et al., Ultramicroscopy 102 (2005) 287.

[5] K. Thompson et al., Ultramicroscopy 107 (2006) 131.

[6] T. F Kelly et al., submitted to Ann. Rev. Mat. Res..

[7] D. Gerthsen et al., phys. stat. sol. (a) 177 (2000) 145.

[8] T. M. Smeeton et al., Appl. Phys. Lett. 83 (2003) 5419.

[9] M. J. Galtrey et al., Appl. Phys. Lett. 90 (2007) in press.

[10] X.Z. Liao et al., Ultramicroscopy 98 (2004) 239. 